\title{
Simplified Voronoi diagrams for motion planning of quadratically-solvable Gough-Stewart platforms
}

Rubén Vaca, Joan Aranda, and Federico Thomas

\begin{abstract}
The obstacles in Configuration Space of quadratically-solvable GoughStewart platforms, due to both kinematic singularities and collisions, can be uniformly represented by a Boolean combination of signs of $4 \times 4$ determinants involving the homogeneous coordinates of sets of four points. This Boolean combination induces a measure of distance to obstacles in Configuration Space from which a simplified Voronoi diagram can be derived. Contrary to what happens with standard Voronoi diagrams, this diagram is no longer a strong deformation retract of free space but, as Canny proved in 1987, it is still complete for motion planning. Its main advantage is that it has lower algebraic complexity than standard Voronoi diagrams based on the Euclidean metric.
\end{abstract}

Key words: Gough-Stewart platform, pure condition, Voronoi diagrams, path planning.

\section{Introduction}

Gough-Stewart platforms whose assembly modes can be obtained by solving only quadratic equations are said to be quadratically-solvable. This family of parallel platforms is defined by certain geometric constraints in the location of their leg attachments to the fixed base and/or moving platform such as coincidence and collinearity (see Fig. 1), or, in general, certain algebraic relations between the coor-

Rubén Vaca and Joan Aranda

Department of Automatic Control and Computing Engineering (UPC), Edifici Vertex, 08034

Barcelona, Spain e-mail: ruben.hernando.vaca|joan.aranda@upc.edu

Federico Thomas

Institut de Robòtica i Informàtica Industrial (CSIC-UPC), Llorens Artigas 4-6, 08028 Barcelona, Spain e-mail: fthomas@iri.upc.edu 

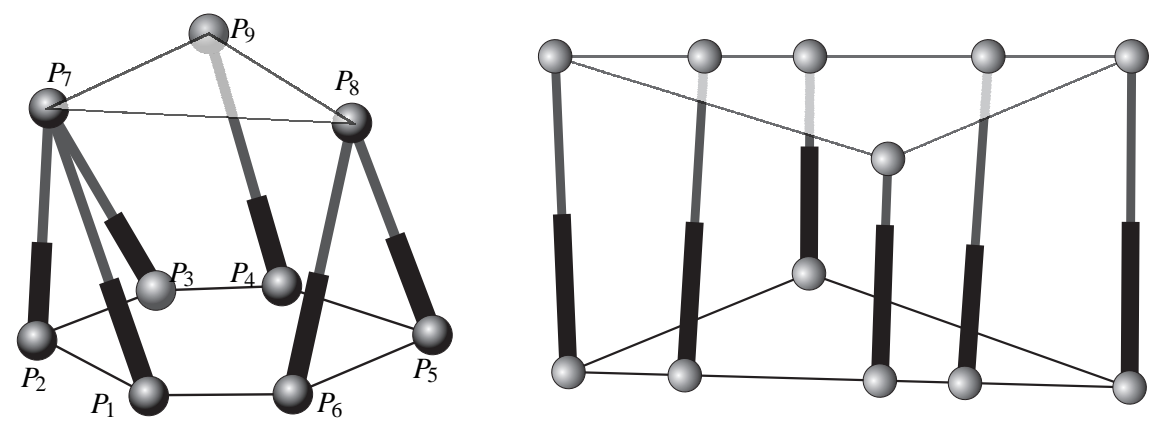

Fig. 1 Examples of two well-known quadratically-solvable Gough-Stewart platforms. Left: the 3-2-1 platform. Right: the Zhang-Song platform.

dinates of the attachments in their local reference frames (see [1] for a non-trivial example).

It is interesting to observe how, when all the elements of a quadratically-solvable Gough-Stewart platform and its environment are approximated by polyhedra, the obstacles in its configuration space, due to both kinematic singularities and collisions, can be uniformly represented by a Boolean combination of signs of $4 \times 4$ determinants involving the homogeneous coordinates of sets of four points. This Boolean combination induces a measure of distance to obstacles from which a simplified Voronoi diagram can be derived. A detailed explanation of all these facts requires a lot of mathematical details but, due to space limitations, we have opted for an informal style to convey the main concepts.

This paper is organized as follows. Section 2 explains how the kinematic singularities of quadratically-solvable Gough-Stewart platforms can always be interpreted as degeneracies of a set of tetrahedra. Section 3 deals with the collision detection between arbitrary polyhedra. Then, it is shown how the regions where either collisions or singularities occur can be uniformly represented by a Boolean combination of signs of $4 \times 4$ determinants. How this representation induces a measure of distance to these forbidden regions is explained in Section 4 and how a simplified Voronoi diagram is derived, in Section 5. A simple planar case is analyzed in Section 6. Finally, we conclude in Section 7.

\section{Singularities of quadratically-solvable parallel robots}

The singularities of quadratically-solvable Gough-Stewart obey a neat algebraic structure. The pure condition of this kind of platforms factorizes into $4 \times 4$ determinants which involve the homogeneous coordinates of sets of four attachments [3]. When the coordinates of the leg attachments in the moving platform are expressed in terms of the position and orientation of the platform, $\mathbf{x}$ and $\Theta$ respectively, three kinds of determinants arise: 
- Plane-Point determinants: They involve three attachments of the fixed base and one of the moving platform. They vanish if, and only if, the point lies on the plane defined by the three points fixed to the base.

- Point-Plane determinants: They involve three attachments of the moving platform and one of the fixed base. The vanish if, and only if, the fixed point lies on the moving plane defined by the three points attached to the platform.

- Line-Line determinants: They involve two attachments of the base and two of the moving platform. They vanish if, and only if, the lines defined by the two couples of points lie on the same plane.

Hence, the singularities of quadratically-solvable platforms can be geometrically interpreted as the degeneracy of tetrahedra. For example, the pure condition of the robot in Fig. 1(left) can be expressed as:

$$
\left[\mathbf{p}_{1} \mathbf{p}_{2} \mathbf{p}_{3} \mathbf{p}_{7}\right]\left[\mathbf{p}_{5} \mathbf{p}_{6} \mathbf{p}_{7} \mathbf{p}_{8}\right]\left[\mathbf{p}_{4} \mathbf{p}_{7} \mathbf{p}_{8} \mathbf{p}_{9}\right]=0
$$

where $\mathbf{p}_{i}$ stand for the homogenous coordinates of leg attachment $P_{i}$ in the world reference frame. Then, this robot is in a singularity if any of the three determinants vanish (i.e., if any of the three associated tetrahedra degenerate). Then, if we define the predicate

$$
\triangle(i, j, k, l)=\left\{\begin{array}{l}
\text { true if }\left[\mathbf{p}_{i} \mathbf{p}_{j} \mathbf{p}_{k} \mathbf{p}_{l}\right]>0, \\
\text { false otherwise, }
\end{array}\right.
$$

the robot's singularity-free region is the set of configurations where

$$
(\triangle(1,2,3,7) \vee \overline{\triangle(1,2,3,7)}) \wedge(\triangle(5,6,7,8) \vee \overline{\triangle(5,6,7,8)}) \wedge(\triangle(4,7,8,9) \vee \overline{\triangle(4,7,8,9)})
$$

is true. This boolean expression can be seen as a shallow (depth 2) AND-OR tree with an AND node at the root. The interest of expressing singularity-free regions in this apparently awkward way becomes obvious when integrating them with collision-free regions as described next.

\section{Basic contacts between polyhedra}

The configuration space obstacle for a pair of polyhedra is the set of configurations at which the two polyhedra overlap. The overlap predicates between polyhedra are logical combinations of primitive predicates. These primitive predicates correspond to the three basic types of contacts between polyhedra [7], which in turn can be expressed using the same three kinds of determinants already used to characterize the singularities of quadratically-solvable platforms.

The formulation of polyhedral interference detection as the evaluation of a boolean formula that depends only on the features (vertices, edges, and faces) of polyhedra with convex faces was proposed by Canny [5]. This test was later generalized, and fully expressed in terms of signs of $4 \times 4$ determinants, by Thomas and Torras [9]. 
As a simple example of boolean formulas for overlap detection, let us consider the segment defined by $P_{1} P_{2}$ and the triangle defined by $P_{3} P_{4} P_{5}$ in $3 \mathrm{D}$. Then, the segment is intersecting the triangle if

$$
\begin{aligned}
(\triangle(1,3,4,5) \otimes \triangle(2,3,4,5)) \wedge & {[(\triangle(1,2,3,4) \wedge \triangle(1,2,4,5) \wedge \triangle(1,2,5,3))} \\
& \vee(\overline{\triangle(1,2,3,4)} \wedge \overline{\triangle(1,2,4,5)} \wedge \overline{\triangle(1,2,5,3))}]
\end{aligned}
$$

is true, $\otimes$ being the exclusive or operator defined as $(a \otimes b)=(a \wedge \bar{b}) \vee(\bar{a} \wedge b)$.

Then, the predicates that define collision-free regions can also be represented as AND-OR trees that can be fused in a seamless way with those representing singularity-free regions through an AND node. The resulting trees can be obviously organized in different ways for higher efficiency using the properties of boolean algebra. One of the overlap predicates for polyhedra with convex faces given in [5] generate a shallow (depth 2) AND-OR predicate tree, whose root is an OR node. Nevertheless, as Canny already observed, it is computationally advantageous to make the predicate tree as deep as possible and it is also desirable for the root to be an AND node.

\section{A measure of distance to obstacles in Configuration Space}

Following the discussion in [4], we can now observe that by letting positive real values represent logical one, and non-positive values represent logical zero, that the min function implements logical AND, and the max function implements logical OR. Thus, for example, the predicate (2) that characterizes the obstacle in configuration space due to kinematic singularities of the robot in Fig. 1 can be expressed as:

$$
\begin{aligned}
\rho(\mathbf{x}, \Theta)=\min ( & \max \left(\left[\mathbf{p}_{1} \mathbf{p}_{2} \mathbf{p}_{3} \mathbf{p}_{7}\right],-\left[\mathbf{p}_{1} \mathbf{p}_{2} \mathbf{p}_{3} \mathbf{p}_{7}\right]\right), \\
& \max \left(\left[\mathbf{p}_{5} \mathbf{p}_{6} \mathbf{p}_{7} \mathbf{p}_{8}\right],-\left[\mathbf{p}_{5} \mathbf{p}_{6} \mathbf{p}_{7} \mathbf{p}_{8}\right]\right), \\
& \left.\max \left(\left[\mathbf{p}_{4} \mathbf{p}_{7} \mathbf{p}_{8} \mathbf{p}_{9}\right],-\left[\mathbf{p}_{4} \mathbf{p}_{7} \mathbf{p}_{8} \mathbf{p}_{9}\right]\right)\right)
\end{aligned}
$$

The above quantity can be used as a measure of distance - it is not a true metric - to the robot's kinematic singularities, because it varies continuously through configuration space. Thus, the translation from a predicate representing the singularity and collision-free regions to a measure of distance to forbidden regions is straightforward. It will be positive at configurations outside them and negative at configurations inside them. Clearly, this measure of distance decomposes the robot's configuration space into regions in which one of the involved determinants is critical in determining the value of $\rho$, that is, small changes in its value cause identical changes in the value of $\rho$. The boundaries between these regions can be seen as a Voronoi diagram. Then, searching the configuration space for singularity and collision-free paths can be reduced to a search on this diagram as explained in the next section. 


\section{Simplified Voronoi diagrams}

A Voronoi diagram is defined to be the set of points equidistant from two or more generators (points, segments, polygons, ...) under the appropriate metric (usually the Euclidean distance). This construction received considerable attention in the early eighties as a useful tool for motion planning (see the textbook of Schwartz and Yap [8], and the references therein, for an introduction and review of the use of Voronoi diagrams in motion planning).

The main advantage of using the Voronoi diagrams based on the measure of distance described in the previous section is that they have a lower algebraic complexity than those resulting from using the Euclidean distance. These diagrams are piecewise linear for fixed orientations of the moving platform while standard Voronoi diagrams would contain quadratic sheets. These simplified Voronoi diagrams are sometimes also called straight skeletons [6]. Despite this important simplification, they still have an important property: any path through free space which starts and ends on the diagram can be continuously deformed so that it lies entirely on the diagram [4]. Thus, they are complete for motion planning, i.e., searching the original space for paths can be reduced to a search on the diagram.

Now, to find a path between two points in free space, it suffices to find a path for each point onto the diagram, and to join these points with a path that lies wholly on the diagram.

\section{Example}

The ideas presented above are for spatial parallel robots. Nevertheless, to exemplify them, we will rely on a planar case because the corresponding configuration space is three-dimensional and hence easily representable.

Let us consider a planar robot whose base is determined by the segment $P_{1} P_{2}$, its moving platform by the segment $P_{3} P_{4}$, and its three legs by the segments $P_{1} P_{3}, P_{2} P_{3}$, and $P_{2} P_{4}$. Let us also assume that the homogeneous coordinates of these points are given by $\mathbf{p}_{1}=(0,0,1)^{t}, \mathbf{p}_{2}=(15,0,1)^{t}, \mathbf{p}_{3}=(x, y, 1)^{t}$, and $\mathbf{p}_{4}=$ $(x+10 \cos (\phi), y+10 \sin (\phi), 1)^{t}$. Therefore, the configuration of the moving platform is determined by $(x, y, \phi)$. We also introduce a boundary region determined by the square $P_{5} P_{6} P_{7} P_{8}$ whose vertex coordinates are $\mathbf{p}_{5}=(-25,40,1)^{t}, \mathbf{p}_{6}=$ $(-25,-40,1)^{t}, \mathbf{p}_{7}=(55,-40,1)^{t}$, and $\mathbf{p}_{8}=(55,40,1)^{t}$.

In this planar case, the regions free from singularities and collisions can be represented by a Boolean combination of signs of $3 \times 3$ determinants. Actually, the region free from kinematic singularities is defined by the predicate

$$
\mathscr{P}_{1}=(\triangle(1,2,3) \vee \overline{\triangle(1,2,3)}) \wedge(\triangle(2,3,4) \vee \overline{\triangle(2,3,4)}),
$$

and the region free from collisions between the moving platform, the base, and the four segments defining the boundary, by the predicate 


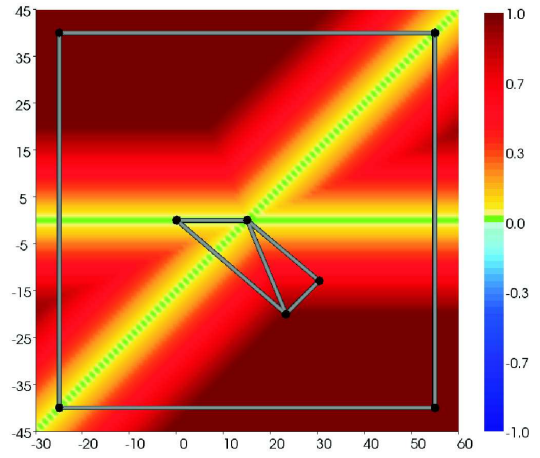

(a)

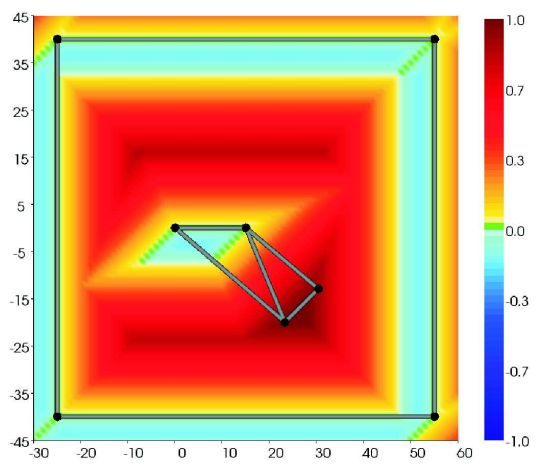

(c)

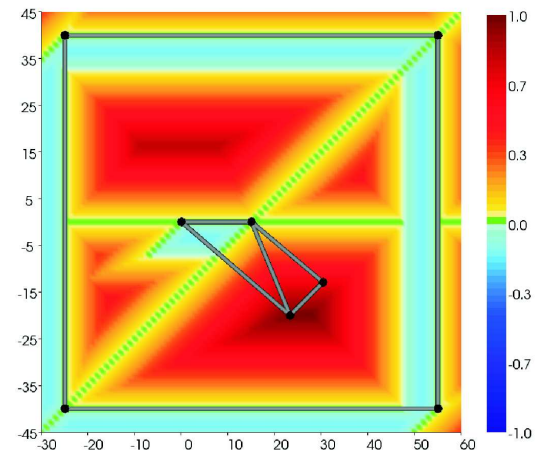

(e)

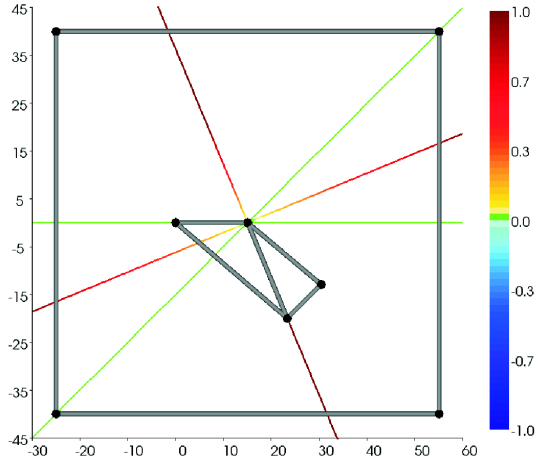

(b)

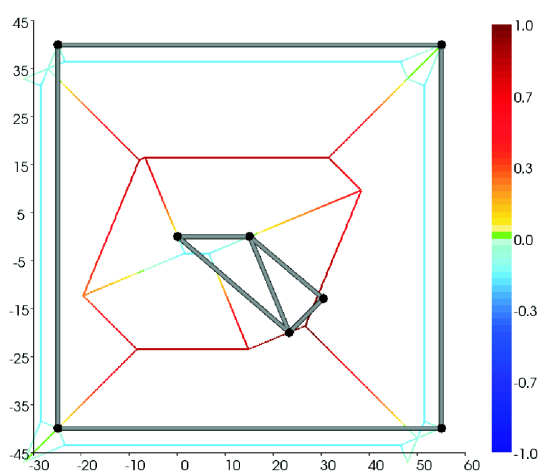

(d)

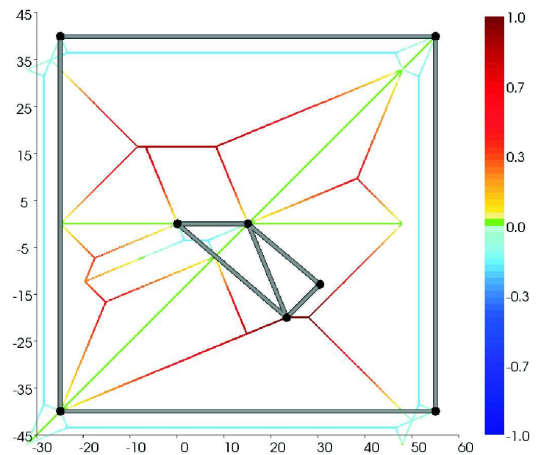

(f)

Fig. 2 Measure of distance to the kinematic singularities (a), and the corresponding Voronoi diagram (b). Measure of distance to the regions where collisions occur (c), and the corresponding Voronoi diagram (d). Measure of distance taking into account both singularities and collisions (e), and the corresponding Voronoi diagram (f). All images are slices of the robot's configuration space for $\phi=\frac{\pi}{4}$. 


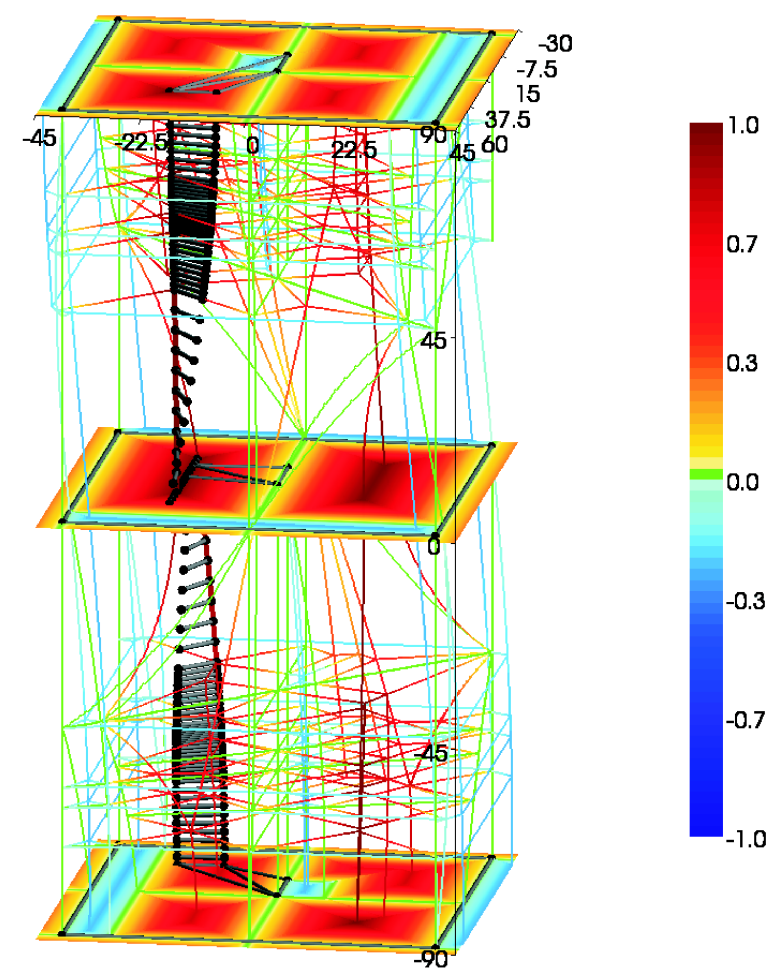

Fig. 3 Representation of the analyzed robot's configuration space in the region defined by $(x, y, \phi)=([-30,60],[-45,45],[-\pi / 2, \pi / 2])$. The plot shows the measure of distance associated with $\mathscr{P}_{1} \wedge \mathscr{P}_{2}$ for $\phi=-\pi / 2,0$, and $\pi / 2$, and slices of the corresponding Voronoi diagram for values of $\phi$ where there is a change in its topology. The lines connecting them correspond to the edges of the Voronoi diagram.

$$
\begin{aligned}
\mathscr{P}_{2} & =\overline{(\triangle(1,2,3) \otimes \triangle(1,2,4)) \wedge(\triangle(1,3,4) \otimes \triangle(2,3,4))} \\
& \wedge \overline{(\triangle(5,6,3) \otimes \triangle(5,6,4)) \wedge(\triangle(5,3,4) \otimes \triangle(6,3,4))} \\
& \wedge \overline{(\triangle(6,7,3) \otimes \triangle(6,7,4)) \wedge(\triangle(6,3,4) \otimes \triangle(7,3,4))} \\
& \wedge \overline{(\triangle(7,8,3) \otimes \triangle(7,8,4)) \wedge(\triangle(7,3,4) \otimes \triangle(8,3,4))} \\
& \wedge \overline{(\triangle(8,5,3) \otimes \triangle(8,5,4)) \wedge(\triangle(8,3,4) \otimes \triangle(5,3,4))}
\end{aligned}
$$

Figs. 2(a) and 2(b) show the measure of distance associated with $\mathscr{P}_{1}$ and its corresponding Voronoi diagram, respectively. Figs. 2(c) and 2(d) show the same information for $\mathscr{P}_{2}$. These Voronoi diagrams represent the regions free from kinematic singularities and free from collisions, respectively. Figs. 2(e) and 2(f) show the measure of distance associated with $\mathscr{P}_{1} \wedge \mathscr{P}_{2}$ and the corresponding Voronoi di- 
agram thus integrating all the information in a single diagram. Finally, Fig. 3 shows how this diagram evolves, as $\phi$ varies, and a singularity and collision-free path connecting the configuration $(35,-20, \pi / 2)$ and $(-10,-15,-\pi / 2)$. Observe how all slices for fixed orientations are arrangements of straight line segments.

\section{Conclusion}

The use of Voronoi diagrams for motion planning received a lot of attention in the eighties. This interest progressively decreased in favor of algorithms based on random samplings. Nevertheless, it still remains as a fundamental tool for those applications in which an exact representation of free space is required. This paper shows how a simplified Voronoi diagram of the singularity and collision-free regions of any quadratically-solvable Gough-Stewart platform can be obtained assuming that the robot itself and its environment can be well-approximated by sets of polyhedra. The result is an elegant algorithm fully expressed in terms of $4 \times 4$ determinants.

Finally, concerning computational efficiency, it is worth noting that many basic geometric tests other than interference detection such as classification, containment, and depth priority tests can be performed by computing sets of determinants. This has motivated the search for efficient determinant computations using hardware implementations such as the triangle processor and its successor, the polygon engine [10]. This is a point that could be explored if the presented ideas should be implemented for complex Gough-Stewart platforms in cluttered environments.

\section{References}

1. Borràs, J., Thomas, F., Torras, C.: On Delta transforms. IEEE Transactions on Robotics, 25(6), 1225-1236 (2009)

2. Borràs, J., Thomas, F., Torras, C.: A family of quadratically-solvable 5-SPU parallel robots. Proc. of the 2010 IEEE Int. Conf. on Robotics and Automation, 4703-4708 (2010)

3. Borràs, J., Thomas, F.: On the primal and dual forms of the Stewart platform pure condition. submitted

4. Canny, J., Donald, B.: Simplified Voronoi diagrams. Discrete and Computational Geometry 3(3), 219-236 (1988)

5. Canny, J.: The Complexity of Robot Motion Planning, MIT Press, Cambridge, Mass. (1987)

6. Eppstein, D., Erickson, J.: Raising roofs, crashing cycles, and playing pool: Applications of a data structure for finding pairwise interactions. Discrete and Computational Geometry 22(4) 569-592 (1999)

7. Lozano-Pérez, T.: Spatial planning: a configuration space approach. IEEE Trans. on Computers C-32(2), 108-120 (1983)

8. Schwartz, J., Yap, C.K.: Advances in Robotics. Lawrence Erlbaum Associates, New Jersey (1986)

9. Thomas, F., Torras, C.: A projectively invariant intersection test for polyhedra. The Visual Computer 18(7), 405-414 (2002)

10. Yamaguchi, F., Tsuda, S., Nagasaki, T.: Applications of the $4 \times 4$ determinant method and the polygon engine. The Visual Computer, 4(4), 176-187 (1988) 